\title{
Integrated, Feed-Forward Hybrid Electric Vehicle Simulation in SIMULINK and its Use for Power Management Studies
}

\author{
Chan-Chiao Lin, Zoran Filipi, Yongsheng Wang, Loucas Louca, \\ Huei Peng, Dennis Assanis, Jeffrey Stein \\ Automotive Research Center \\ The University of Michigan
}

Copyright (C) 2001 Society of Automotive Engineers, Inc.

\begin{abstract}
A hybrid electric vehicle simulation tool (HE-VESIM) has been developed at the Automotive Research Center of the University of Michigan to study the fuel economy potential of hybrid military/civilian trucks. In this paper, the fundamental architecture of the feed-forward parallel hybrid-electric vehicle system is described, together with dynamic equations and basic features of sub-system modules. Two vehicle-level power management control algorithms are assessed, a rule-based algorithm, which mainly explores engine efficiency in an intuitive manner, and a dynamic-programming optimization algorithm. Simulation results over the urban driving cycle demonstrate the potential of the selected hybrid system to significantly improve vehicle fuel economy, the improvement being greater when the dynamicprogramming power management algorithm is applied.
\end{abstract}

\section{INTRODUCTION}

Growing environmental concerns coupled with concerns about global crude oil supplies stimulate research aimed at new, fuel-efficient vehicle technologies. Hybrid-electric vehicles (HEV) appear to be one of the most viable technologies with significant potential to reduce fuel consumption within realistic economical, infrastructural and customer acceptance constraints. Dozens of prototype/concept hybrid vehicles have been developed. Toyota and Honda have already launched production vehicles and many other major automakers are expected to launch hybrid vehicles in the next 3-5 years. Due to the existence of dual power-sources, the additional design degrees of freedom of HEV offer unprecedented possibilities in fuel economy and exhaust emissions, particularly if parallel powertrain architectures are employed. At the same time, the complexity of the new vehicle system requires the application of simulations for accurate sizing and matching studies, as well as for development of control algorithms well ahead of the final design and physical prototyping.

Most of the control strategies developed for parallel HEV fall into three categories. The first type applies intelligent control techniques such as rules/fuzzy logic/NN for estimation as well as control algorithm development [1 and 2]. The second type of approach is based on static optimization methods. Commonly, to calculate the cost of energy, the electric energy is translated into an equivalent amount of fuel [3 and 4]. The optimization scheme then figures out proper energy and/or power split between the two energy sources under steady-state operations. Due to its relatively simple point-wise optimization nature, it is possible to extend the optimization scheme to solve the simultaneous fuel economy and emission optimization problem [5]. The basic idea of the third type of HEV control algorithm is similar to that of static optimization; however, the optimization was performed for dynamic systems [6]. Further, the optimization is with respect to a time horizon, rather than for a fixed point in time. In general, the power split algorithm from the dynamic optimization will be more accurate under transient conditions. Usually, the dynamic optimization algorithms are not implementable due to their preview nature and heavy computation requirement. They are, however, a good benchmark based on which the first two types of algorithms can be improved or compared against.

The objective of this work is to develop an integrated hybrid vehicle simulation tool and use it for the design of 
energy management control algorithms. The basis for our Hybrid Vehicle-Engine SIMulation (HE-VESIM) is the high-fidelity conventional vehicle simulator VESIM previously developed at the University of Michigan [7]. VESIM has been validated against measurements for a Class VI truck, and proven to be a very versatile tool for mobility, fuel economy and drivability studies. To construct a hybrid-vehicle simulator, some of the main modules require modifications, e.g. the engine needs to be reduced in size/power, and the electric component models need to be created and integrated into the system. Our HEV simulation effort will focus on parallel post-transmission configurations, where the electric motor is mechanically coupled to the output shaft. A feed-forward simulation scheme will be employed so as to enable studies of control strategies under realistic transient conditions. The integrated HEV simulation will be implemented in SIMULINK to allow for easy reconfiguration of the system and to enable the designer to select proper models depending on specific simulation goals. Two control algorithms are investigated in this paper: a rule-based and a dynamic programming optimization algorithm.

The paper is arranged as follows. The configuration of the newly developed hybrid electric vehicle system in SIMULINK is discussed first, followed by the description of features of the main simulation modules: diesel engine, drivetrain, vehicle dynamics and electric components. Next, two power management algorithms: a rule-based algorithm, and a dynamic programming based optimization algorithm are introduced. The complete hybrid vehicle simulation is then used to assess the acceleration ability and the fuel economy of the hybrid vehicle through comparisons with its conventional counterpart. The two control strategies are evaluated through simulation predictions of fuel consumption over a driving cycle, followed by the summary and conclusions.

\section{HYBRID-ELECTRIC VEHICLE SYSTEM}

The vehicle system considered in this work is a $4 \mathrm{X} 2$ Class VI truck configured as a parallel hybrid with the electric motor positioned after the transmission. The schematic of the vehicle and the propulsion system is given in Figure 1. The engine is connected to the torque

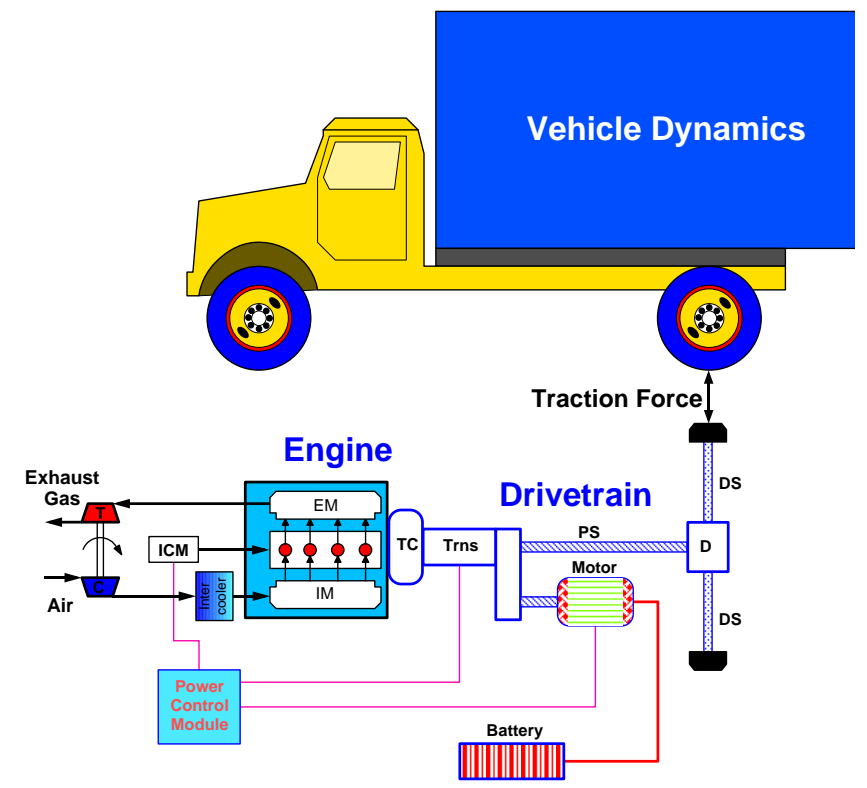

Figure 1: Schematic of the integrated vehicle system.

converter (TC), whose output shaft is then coupled to the transmission (Trns). The coupling at the transmission output side engages or disengages the electric motor depending on the operation mode of the hybrid. Hence,
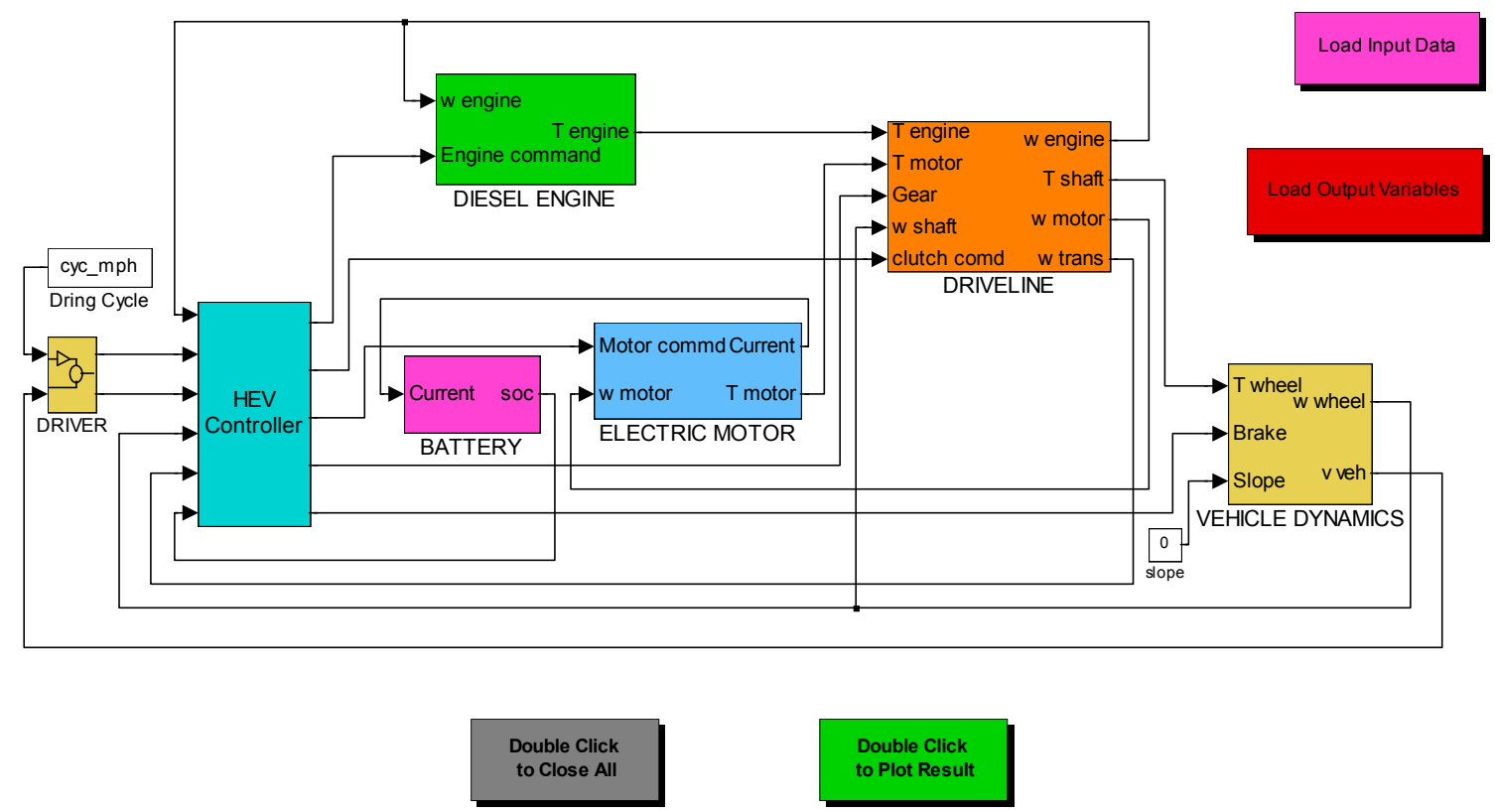

Figure 2 Hybrid-electric vehicle simulation in SIMULINK 
the transmission and/or electric motor can be linked to the propeller shaft (PS), differential (D) and two driveshafts (DS), coupling the differential with the driven wheels.

The complete vehicle system simulation is structured to directly resemble the layout of the physical system. In order to have a high degree of flexibility, the simulation structure is implemented in the MATLAB/SIMULINK graphical software environment, as shown in Figure 2. Links between main modules represent the physical parameters that actually define the interaction between the components, such as shaft torque and angular velocity, or electrical current and voltage. The HEV controller contains the power management logic and sends control signals to the components modules based on the feedback about current operating conditions. Finally, a "driver" module allows the feed-forward simulation to follow a prescribed vehicle speed schedule. The Intelligent Speed Controller (IVS) fulfills that role and provides the driver demand signal and braking based on the specified speed setting and the current vehicle speed.

\section{ENGINE}

The engine model is derived from the high fidelity, thermodynamic engine system previously developed for the conventional vehicle [7 and 11]. The high fidelity engine model was comprised of multiple cylinder modules linked with external component modules for manifolds, compressors and turbines, heat exchangers, air filters, and exhaust system elements. In order to support the computationally intensive simulations over long driving cycles and facilitate easy scaling of the engine, the thermodynamic engine model is replaced by a look-up table that provides brake torque as a function of instantaneous engine speed and mass of fuel injected per cylinder/cycle. The look-up table is actually generated using a previously validated high fidelity engine system code [11], hence it is possible to physically vary the size of the engine, or its design, and have a realistic representation of the effect of a given change. For the parallel hybrid application, the original V8 $7.3 \mathrm{~L}$ diesel is downsized by reducing the number of cylinders to 6 , and hence the displacement to $5.5 \mathrm{~L}$. The turbomachinery maps are scaled to match the smaller engine, following the methodology described in [20]. The whole procedure for generating torque look-up tables based on predictions of a validated high fidelity engine system code is illustrated in Figure 3 . The specifications of both the V8 engine for the conventional vehicle and the $\mathrm{V} 6$ engine for the hybrid application are given in Table 1 of the Appendix.

In order to retain features of the engine system critical for the transient response, the complete fuel control logic is retained in the look-up table based model, as shown in Figure 4. The diesel engine fuel injection controller provides the signal for the mass of fuel injected per cycle based on driver demand, supplied by the IVS (driver) module, environmental conditions and current engine operating conditions, i.e. engine speed and boost pressure. The instantaneous engine speed is provided as the output of the engine dynamics block (Figure 4), while the nominal value of boost pressure is tabulated as the function of speed and load based on predictions of the high fidelity code. Hence, the part of the fuel control logic that limits the fuel at low boost is retained in a meaningful way. In addition, a carefully calibrated time delay is built-in to represent the effect of turbo-lag on transient response to rapid increases of engine "rack positions". More details about the engine subsystem and the fuel controller are provided in the work by Assanis et al. [7].

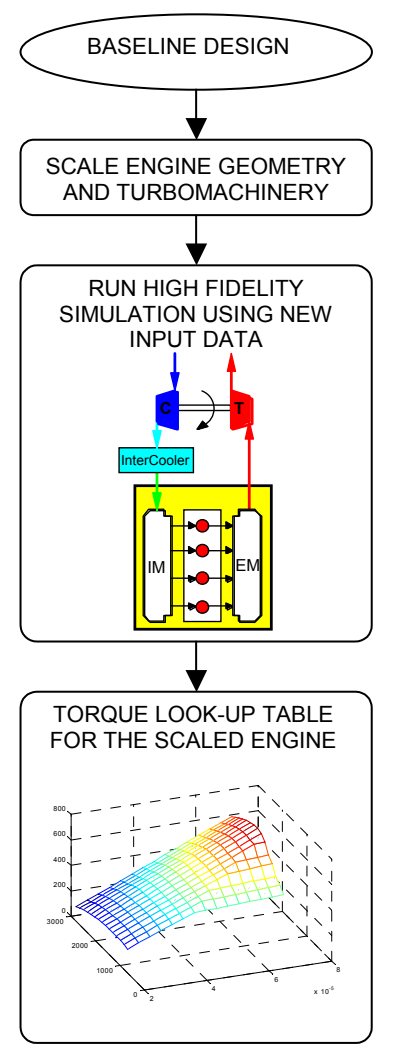

Figure 3: Generating a torque map for a scaled engine

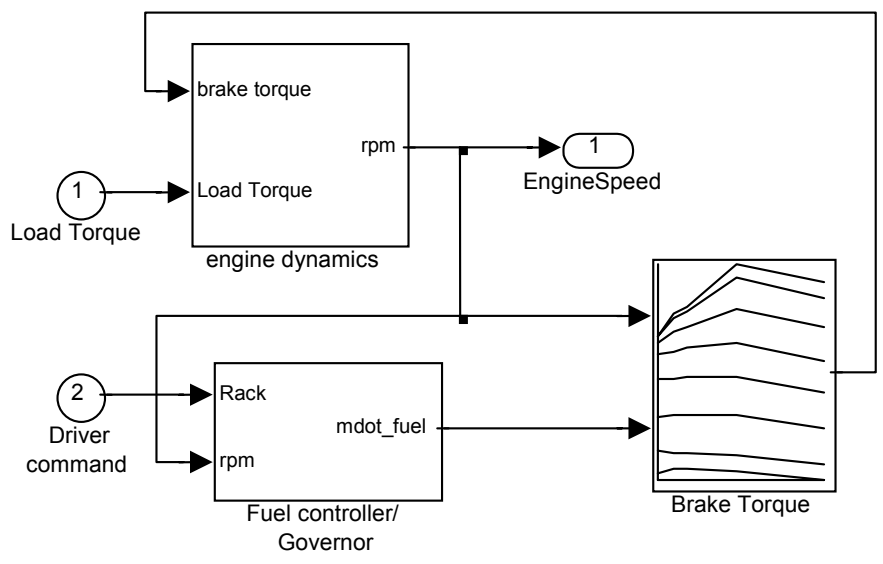

Figure 4: Engine subsystem in SIMULINK 


\section{DRIVETRAIN}

The driveline module consists of the torque converter, transmission, propshafts, differential, and drive shafts. It provides the connection between the engine, the electric motor and the vehicle dynamics module (see Figure 1). The torque converter input shaft connects to the engine flywheel. On the other end, the transmission-out shaft and/or the electric motor shaft are connected to the propeller shaft, the differential and, via driveshafts, to the wheels. Hence, the connection between the driveline and the vehicle dynamics model occurs at the wheel. The drivetrain model is constructed using the bond graph modeling language [12 and 13] and implemented in the 20SIM system-modeling environment [14]. The bond graph language is selected due to its capability of effortless generation of models with different complexity [15]. The detailed development of the drivetrain model is described in [16]; its key parameter values are given in Table 2 of the Appendix.

The dynamic behavior of the drivetrain is represented by ordinary differential equations that describe the kinematic and dynamic behavior of the real system. These equations are automatically generated by 20SIM as standard C code. They are then converted into a C-MEX function. Hence, the final product is an Sfunction suitable for direct integration with the SIMULINK model.

\section{VEHICLE DYNAMICS}

The vehicle subsystem includes the wheels/tires, axles, suspensions and body of the vehicle. A number of approaches can be used to model vehicle dynamics depending on the overall simulation objectives. A single Degree of Freedom (DOF), point mass model, can be selected for an initial estimate of vehicle performance as different powertrain options are explored. The model complexity can be enhanced with more DOFs as more severe excitations (road roughness, steering, braking, etc.) are introduced into the model. This is necessary for the investigation of vehicle-powertrain interactions during such extreme transients that induce significant pitch motion. The complexity of the model can be systematically adjusted, as proposed by Louca et. al.[15], to accommodate the needs of a specific scenario.

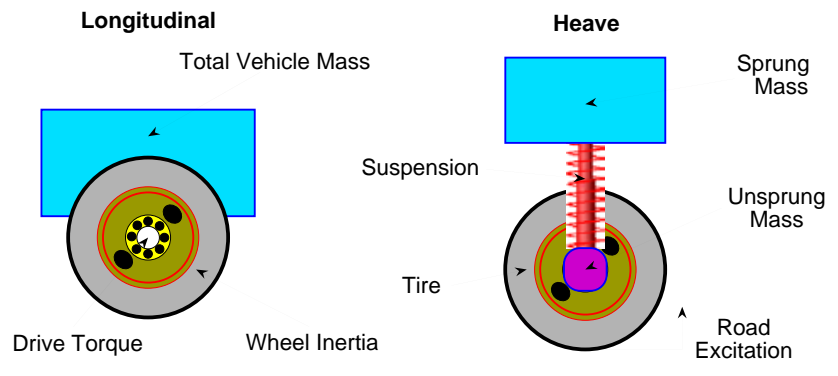

Figure 5: Schematic of vehicle dynamics.
The studies in this work consider only the acceleration of the vehicle on a smooth road where the excitation does not generate significant pitch motion. Therefore, for this "mild" scenario, the enhanced point mass model, shown in Figure 5, adequately predicts the interactions between the powertrain and vehicle dynamics. The model is composed of two components that describe the dynamic behavior of the vehicle in the longitudinal and heave directions. The two components are coupled through the road/tire interaction. The development of the vehicle dynamics model is given in [16] and its key parameter values are given in Table 3 of the Appendix. The vehicle dynamics are also modeled using the bond graph language within the 20SIM environment. The dynamic equations are finally converted into a C-MEX function using the same procedure as in the drivetrain module.

\section{ELECTRIC SUB-SYSTEMS}

Two sub-systems were added to the electric path: a DC motor, and a lead-acid battery. Their characteristics are described in the following.

DC-MOTOR/GENERATOR - Because the engine of the conventional truck is roughly $210 \mathrm{KW}$ and the engine for the hybrid is downsized to about $157 \mathrm{KW}$ (8 cylinders reduced to 6 cylinders), a $49 \mathrm{KW}$ permanent magnet DC motor is selected. The efficiency/loss data, obtained from the Advisor program [9], have the form $\eta_{m}=f\left(T_{m}, \omega_{m}\right)$ (see Figure 6). In other words, the efficiency of the motor is a function of motor torque and motor speed. The motor dynamics are approximated by a first-order lag. However, due to the battery power and motor torque limit, the final motor dynamics assume the following form:

$$
\begin{aligned}
& \text { Positive Motor Torque: } \\
& \qquad T_{m}=\min \left(T_{m_{-} \text {request }}, T_{m_{-} \max }, T_{m_{-} \text {bat }}\right) \cdot \frac{\lambda_{m}}{s+\lambda_{m}}
\end{aligned}
$$

Negative Motor Torque:

$$
T_{m}=\max \left(T_{m_{-} \text {request }}, T_{m_{-} \max }, T_{m_{-} \text {bat }}\right) \cdot \frac{\lambda_{m}}{s+\lambda_{m}}
$$

where $T_{m_{-} \text {request }}$ is the requested motor torque, $T_{m_{-} \max }$ is the maximum torque the motor can generate under current motor speed, $T_{m \text { bat }}$ is the maximum motor torque due to battery constraint, $T_{m}$ is the calculated motor torque, and $\lambda_{m}$ characterizes the motor dynamics, and is the inverse of the motor time constant. The load current for the battery (to be presented below) can then be calculated from the following equation:

$$
i_{t b}= \begin{cases}\frac{\omega_{m} \cdot T_{m} \cdot \eta_{m}}{e_{t b}} & \text { if } T_{m}<0 \\ \frac{\omega_{m} \cdot T_{m}}{e_{t b} \cdot \eta_{m}} & \text { if } T_{m}>0\end{cases}
$$


where $e_{t b}$ is the battery terminal voltage, $\eta_{m}$ is the motor efficiency, and $\omega_{m}$ is the motor speed.

BATTERY - The battery model [8] used is essentially two RC circuits connected in series (see Figure 7). The state dependent internal resistance $\tilde{R}$ and the open circuit voltage $V_{o c}$ are functions of the battery State Of Charge (SOC). The other resistance (the terminal ohmic resistance $R_{b}$ ) and the two capacitances (the polarization capacitance $C_{p}$ and incipient capacitance $C_{i}$ ) are assumed to be constant. The voltages across the two capacitors and the SOC are the three state variables for the battery. Because the two RC-circuit modes are much faster than the dynamics of SOC, in the simplified model (used for dynamic optimizations) we will only have one state for the battery-the SOC.

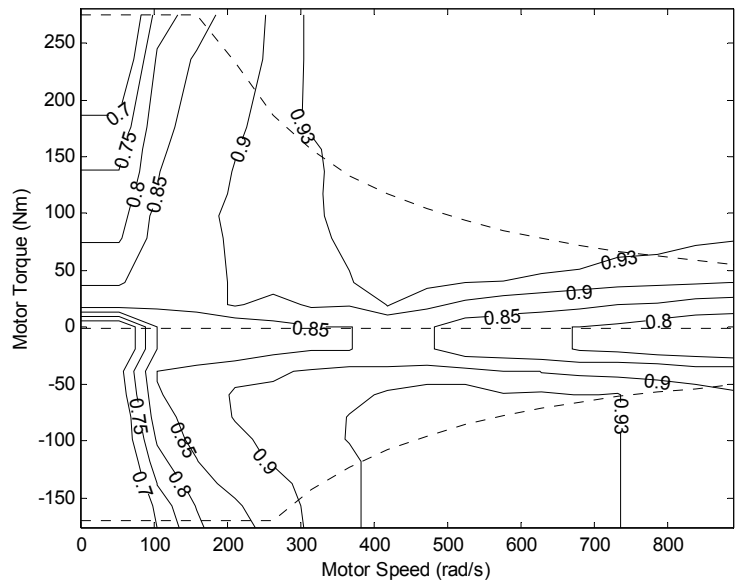

Figure 6: Efficiency map of the DC motor - source [9]

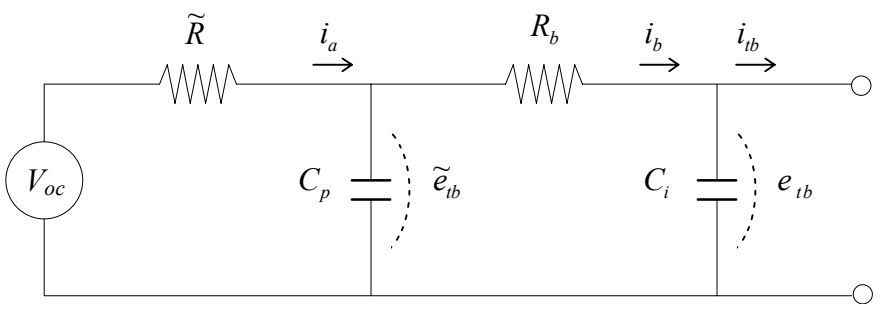

Figure 7: Battery model

In addition to introducing extra dynamics, the battery is also important in the overall HE-VESIM fuel economy because of its nonlinear and non-symmetric efficiency characteristics. Figure 8 shows the charging and discharging efficiency of the battery. This figure was obtained by reducing the dynamic model to a static one and then computing the instantaneous power efficiency. The process of this efficiency calculation can be found in [18]. It can be clearly seen that, overall, the optimal combined charging-discharging efficiency is obtained around $\mathrm{SOC}=0.6$.

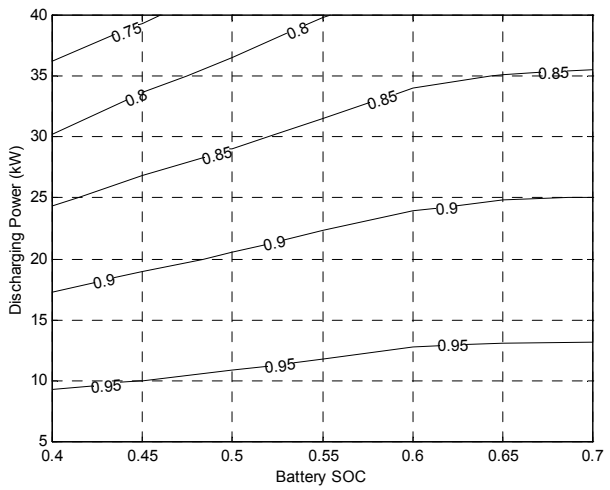

a)

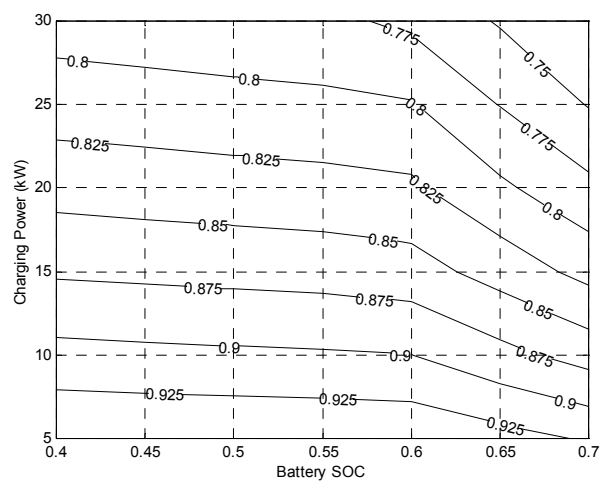

b)

Figure 8: Efficiency maps of the lead acid battery: a) discharging efficiency; b) charging efficiency.

\section{POWER MANAGEMENT ALGORITHMS}

In this section, we will present two power management algorithms: a rule-based algorithm, and a dynamic programming based optimization algorithm. The rule-based algorithm is mainly based on engineering intuition. However, due to the multi-variable nature of HEV control problems, these rules usually fail to capture all the important phenomena. The dynamic programming algorithm can help us understand the deficiency of the rules, and thus makes it possible to construct improved (and more complicated) rules.

RULE BASED ALGORITHM - The rule-based energy management strategy was developed based on engineering intuition and simple analysis of component efficiency tables/charts [10]. The design process starts from interpreting the driver pedal signal as a power request, $P_{r e q}$. According to the power request, an energy management controller determines the power flow in the hybrid powertrain. The operation of this controller can be divided into three modes, each of which is described in the following:

Normal Mode: Based on the engine efficiency map shown in Figure 9, a pre-selected "engine on" power line, $P_{e_{-} o n}$, and "motor assist" power line, $P_{m_{-} a}$, are chosen. If the total power request is less than the "engine on" power level, the electric motor will supply the requested power. Beyond $P_{e_{-} \text {on }}$, the engine replaces the 
motor to provide the total power request. Once the power request exceeds what the engine can efficiently generate, $P_{m_{-} a}$, the motor is activated to supply the additional power $\left(P_{\text {req }}-P_{m_{-} a}\right)$. In this mode, the engine always operates within the high efficient region (between $P_{e_{-} o n}$ and $\left.P_{m_{-} a}\right)$.

Charging Mode: a charge-sustaining strategy is implemented to assure that the battery State Of Charge (SOC) stays within preset upper and lower bounds. The $55-60 \%$ SOC range is chosen for efficient battery operation. When the SOC drops below the low limit $S O C_{\min }$, the energy management controller will switch to the battery recharge mode $\left(\right.$ Flag $_{c h}=$ True $)$. A preselected recharge power level, $P_{c h}$, is added to the driver power request, and the motor power command is forced to become negative to recharge the battery. One exception is that when the total power request is less than the "engine on" power level $\left(P_{\text {tot }}<P_{e_{-} o n}\right)$, the motor will still propel the vehicle to avoid the engine operating in this inefficient region. The battery recharge mode will not stop until the SOC hits the upper bound $S O C_{\text {max }}(60 \%)$.

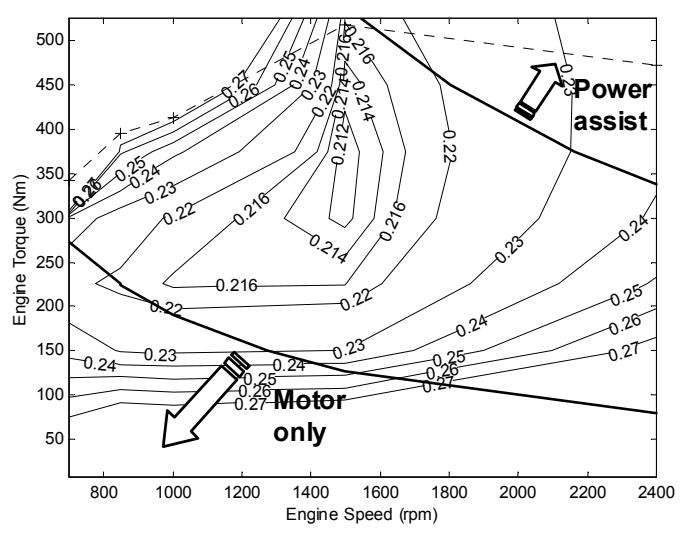

Figure 9: Rule based power management

Braking Mode: When the driver steps on the brake pedal, it is interpreted as a negative power request $\left(P_{r e q}<0\right)$. The regenerative braking is activated to absorb the braking power. However, when the braking power request exceeds the regenerative braking capacity $P_{m_{\text {min }}}$, the hydraulic braking will be activated to assist the vehicle deceleration.

The logic of the rule-based algorithm is summarized in the following:

$$
\begin{aligned}
& \text { IF } S O C<S O C_{\text {min }} \\
& \text { Flag }_{c h}=\text { True }, P_{\text {tot }}=P_{\text {req }}+P_{c h} \\
& \text { IF } S O C>S O C_{\text {max }} \\
& \text { Flag }_{c h}=\text { False }, \quad P_{\text {tot }}=P_{\text {req }}
\end{aligned}
$$

(A) Normal Mode (If Flag $c$ False and $P_{\text {req }}>0$ )

$$
\begin{aligned}
& \text { IF } P_{t o t} \leq P_{e_{-} o n} \\
& P_{e}=0, \quad P_{m}=P_{t o t} \\
& \text { IF } P_{e_{-} o n}<P_{t o t} \leq P_{m_{-} a} \\
& \quad P_{e}=P_{t o t}, \quad P_{m}=0 \\
& \text { IF } P_{m_{-} a}<P_{t o t} \leq P_{m_{-} a}+P_{m_{-} \max } \\
& \quad P_{e}=P_{m_{-} a}, \quad P_{m}=P_{t o t}-P_{m_{-} a} \\
& \text { IF } P_{t o t}>P_{m_{-} a}+P_{m_{-} \max } \\
& P_{e}=P_{t o t}-P_{m_{-} \max }, \quad P_{m}=P_{m_{-} \max }
\end{aligned}
$$

(B) Charging Mode (If Flag $_{c h}=$ True and $P_{r e q}>0$ )

$$
\begin{aligned}
& \text { IF } P_{t o t} \leq P_{e_{-} o n} \\
& \quad P_{e}=0, \quad P_{m}=P_{r e q} \\
& \text { IF } P_{e_{-} o n}<P_{t o t} \leq P_{e_{-} \max } \\
& \quad P_{e}=P_{t o t}, \quad P_{m}=-P_{c h} \\
& \text { IF } P_{t o t}>P_{e_{-} \max } \\
& \quad P_{e}=P_{e_{-} \max }, \quad P_{m}=P_{r e q}-P_{e_{-} \max }
\end{aligned}
$$

(C) Braking Mode (IF $P_{r e q}<0$ )

$$
\begin{aligned}
& \text { IF } P_{r e q} \geq P_{m_{-} \text {min }} \\
& \quad P_{e}=0, \quad P_{m}=P_{r e q}, \quad P_{\mathrm{b}}=0 \\
& \text { IF } P_{r e q}<P_{m_{-} \text {min }} \\
& \quad P_{e}=0, \quad P_{m}=P_{m_{-} \min }, \quad P_{b}=P_{r e q}-P_{m_{-} \min }
\end{aligned}
$$

It should be noted that because it is not straightforward to figure out whether and how the transmission should be shifted in a different manner (from the original shift map designed for the larger, $7.3 \mathrm{~L}$ 8-cylinder engine), we decided to use the same shift logic in the rule-based algorithm.

DYNAMIC PROGRAMMING BASED ALGORITHM As opposed to the rule-based algorithm, the dynamic programming, or similar optimization algorithms, usually rely on a model to compute the best control strategy. The model can be either analytical or numerical; in other words, it can work with numerical black boxes such as HE-VESIM. In the discrete-time format, the model could have the form $x(k+1)=f(x(k), u(k))$. And the goal of the optimization scheme is to minimize a cost function. In this paper, the cost function is assumed to consist of only fuel consumption rate. In the future, when proper emission models are included, the simultaneous fuel economy-emission optimization problem can be solved. The cost function we used has the following form:

$$
J=f u e l=\sum_{k=0}^{N-1} L(x(k), u(k)) \quad(k g)
$$

where in this paper the only term included in the $L$ function is the instantaneous fuel consumption rate. The 
optimization problem is solved under proper inequality constraints to ensure that the engine speed, SOC, fuel consumption and motor torque are all within their corresponding bounds. Also, equality constraints are imposed so that the vehicle always follows the specified driving cycle speed, as well as the acceleration prescribed by the driving cycle. To accelerate computations, pre-computed tables are constructed for grid points for all possible states and control signals. The detailed procedures of the look-up-table based dynamic programming algorithm have been reported in [19] and thus are not repeated here.

It should be noted that a simplified model (with only 2 state variables, SOC and gear number) of the HEVESIM was generated for the dynamic programming algorithm, because the computation time becomes unacceptably long for higher order systems. After the optimal control strategy is found, we then apply the control signals to the original HE-VESIM model (see Figure 2) to ensure that the performance number is obtained from the same (complex) model.

\section{INTEGRATION AND VALIDATION}

The hybrid vehicle system simulation (HE-VESIM) consists of five main modules: engine, driveline, electric motor, battery, and vehicle structure. The interaction between the propulsion modules is in the form of "active" and "resistive" torques, as well as shaft angular speeds. The simulation is configured in a feed-forward manner, where everything starts with the driver action and the "pedal position" signal being sent to the injection system controller. The engine simulation provides as outputs the instantaneous value of engine torque and the rotational speed. The torque undergoes multiple transformations as it is transmitted through the torque converter and the transmission. The final value at the wheel depends on the operating mode, i.e. transmission gear ratio and the contribution of the electric motor/generator, as well as on flexing of the propeller and drive shafts. The torque on the wheels is converted into tractive forces, which in conjunction with other information about the vehicle and the terrain determines vehicle dynamic behavior. Hence, the vehicle dynamics module returns the instantaneous vehicle speed and the wheel angular velocity. This information is propagated back through the system, all the way to the TC output shaft, thus determining the torque converter turbine speed and the speed ratio of the TC. The latter determines the TC pump torque, which is in turn supplied to the engine module as resistive torque. The solution of the engine dynamic equations determines the engine speed value for the next integration step.

The integration of these dynamic modules is performed in the SIMULINK environment. Its graphical programming capabilities allow easy coupling of the modules, as long as each one of them has a desired set of input/output links. However it was known (e.g., [17]) that the flexibility of SIMULINK comes with a certain overhead in terms of computational efficiency, the actual magnitude being strongly dependent on the level of system decomposition and the number of component modules and links. In order to enhance computational efficiency, some of the more complex modules are programmed in C (drivetrain, vehicle dynamics), and configured as self-contained SIMULINK blocks using the MEX function standard.

Prior to the studies of the hybrid-electric system, the simulation of the conventional Class VI truck was validated through comparisons of VESIM predictions with measurements obtained on a real vehicle on International's proving grounds [7]. The comparison of calculated results and measurements for two acceleration tests ( 0 to $60 \mathrm{mph}$ and 30 to $50 \mathrm{mph}$ ) demonstrated very good agreement [7], hence it was concluded that the simulation could be used for further studies of configurations derived from the original one.

\section{SIMULATION RESULTS}

The vehicle studied is the International 4700 series. The diesel engine is downsized from the V8 (7.3L) to a V6 and a $49 \mathrm{KW}$ electric motor is selected to assist the internal combustion engine. The transmission gear ratios are matched according to the demands of the newly-configured parallel hybrid powertrain with the post-transmission motor location. Total vehicle mass is $7258 \mathrm{~kg}$. Basic engine, drivetrain and vehicle specifications are given in the Appendix. The performance of the hybrid vehicle during launch and hard acceleration from 0 to $60 \mathrm{mph}$ is assessed first. Then the virtual hybrid electric truck with the power management controller is tested through simulation over the Federal Urban Driving Schedule in order to evaluate its potential for fuel economy improvement.

ACCELERATION $0-60 \mathrm{mph}$ - The 0 to $60 \mathrm{mph}$ acceleration test was simulated in order to verify that the parallel hybrid with the downsized engine retains the same acceleration performance as the conventional baseline vehicle. The comparison of vehicle speed profiles is shown in Figure 10. The hybrid achieved 60 $\mathrm{mph}$ slightly earlier than the conventional truck, primarily due to better performance immediately after launch. reveals more details about the system response during this rapid transient. A favorably high value of motor torque at very low speeds (see Figure 11b) compensates for the slower response of the diesel engine due to turbo lag (see Figure 11c), hence the combined value results in a higher acceleration at launch. Since the driver power demand is $100 \%$, the motor continuously operates at its highest available torque to assist the engine until the desired speed is achieved. Figure 11a illustrates the cumulative fuel consumption of the conventional truck and the hybrid electric vehicle during the acceleration run. Obviously, the downsized engine consumes significantly less fuel, while it should be kept in mind that part of the energy comes from the battery. 


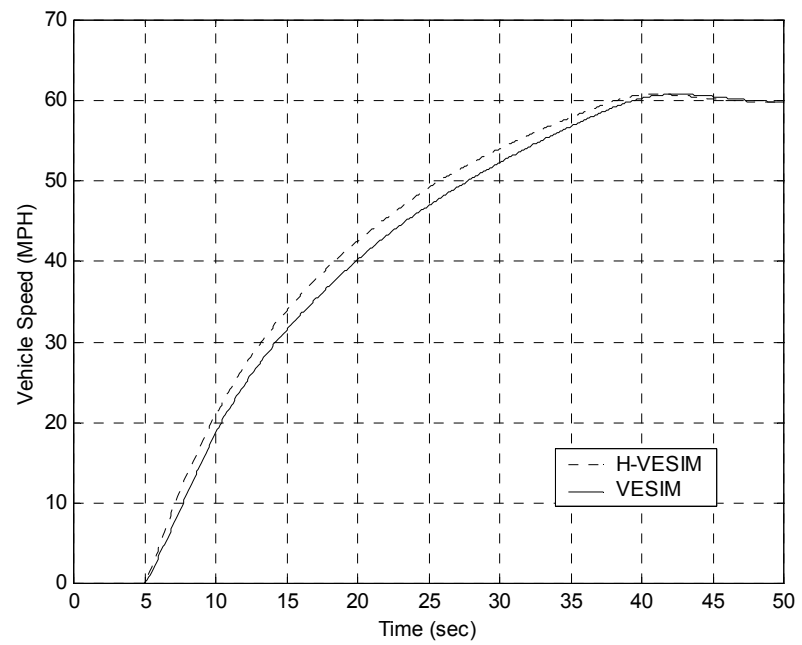

Figure 10: Speed profile comparison of conventional vs. hybrid truck during 0-60 mph acceleration

FUEL ECONOMY OVER A DRIVING CYCLE - The Federal Urban Driving Schedule (FUDS, see Figure 12) was used to evaluate the fuel economy of the delivery truck studied in this work. Once the speed profile of the driving cycle is specified, the corresponding torque at the tires necessary to follow the speed profile is calculated (Figure 12) and used as the desired output for both the rule-based and the optimal algorithms. It is interesting to examine more closely how one of the control algorithms, e.g. the rule-based controller, switches between different modes of hybrid operation during a driving schedule. For that purpose, a close-up of the 430 to 540 second period of the driving cycle is given in Figure 13. This segment of the cycle includes two acceleration-cruisingdeceleration profiles (see Figure 13a), the first one requiring harder acceleration to higher speed. The battery SOC, engine power and electric motor power are given in Figures 13b, c and d, respectively. The truck launches from stop using only the motor to avoid inefficient engine operation under low power demands. However, the engine is turned on very quickly, since the power demand requires the output from both the engine and the electric motor. From 450 to $477 \mathrm{sec}$, the power required to cruise at the speed of $35 \mathrm{mph}$ is less than the "engine on" power level, hence the engine is disengaged and the motor supplies all the torque required at the wheels. When the truck decelerates, the regenerative braking is applied; hence the motor operates as a generator to recover the energy that would otherwise be dissipated in brakes (477 to $490 \mathrm{sec}$ and 530 to $537 \mathrm{sec}$ interval). It should be noted that when the battery SOC hits the lower bound (55\%), at 523 seconds into the schedule, the engine is immediately turned on to power the truck, as well as to recharge the battery. Hence, the electric motor is switched to the generator mode and its torque becomes negative.
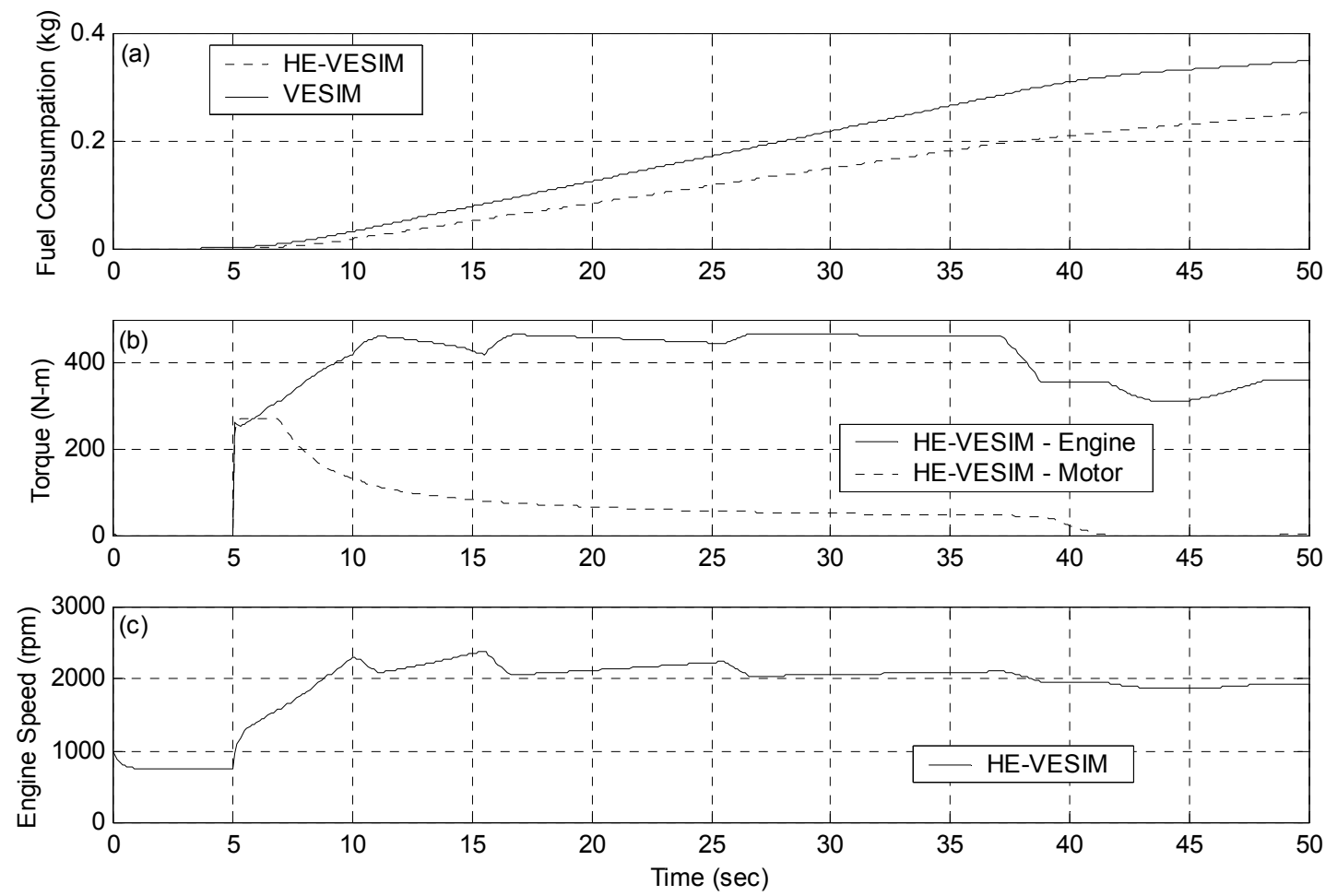

Figure11: Critical system variables during 0-60 mph acceleration: conventional truck (VESIM) vs. the hybrid electric truck (HE-VESIM) 

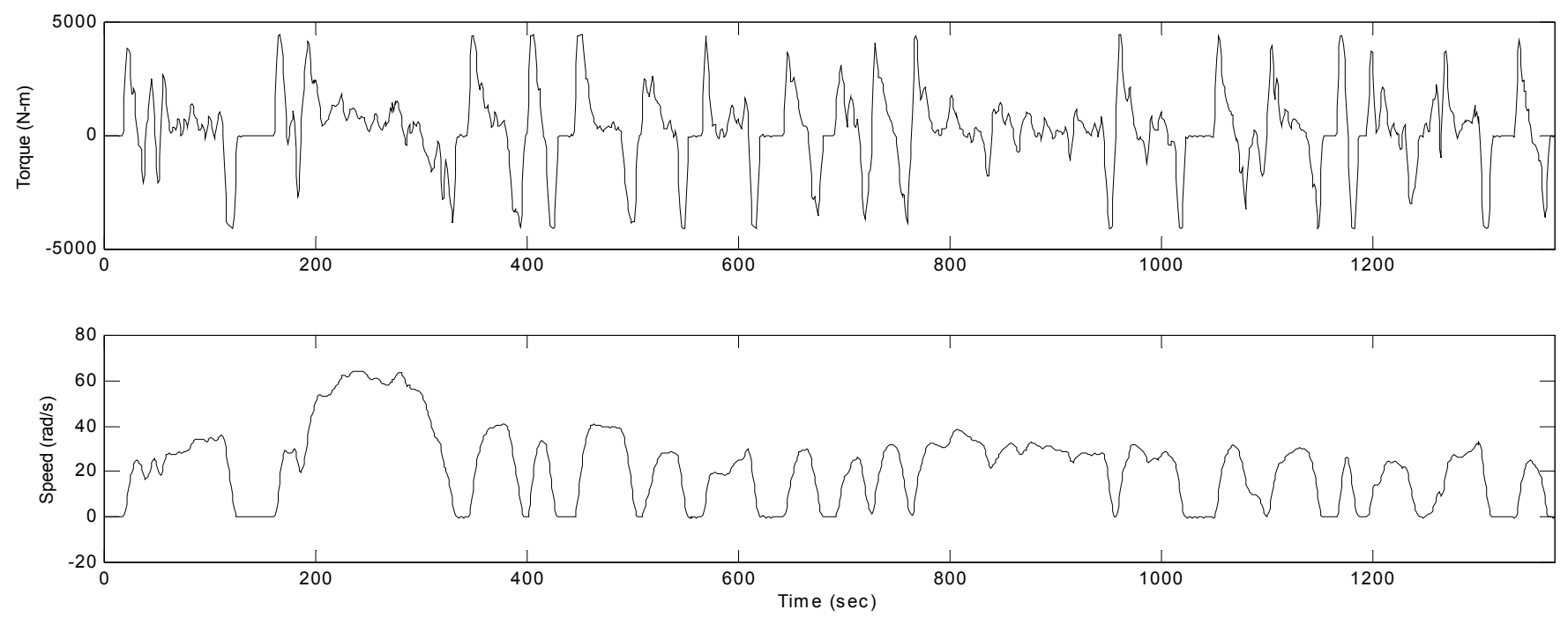

Figure 12: Federal Urban Driving Schedule (FUDS

Furthermore, a close-up examination of the behavior of two vehicles with different control algorithm between 690 to 765 second of the driving cycle is given in Figure 14. The behaviors of the two vehicles under deceleration (from 714 to $726 \mathrm{sec}$, and 749 to $763 \mathrm{sec}$ ) are identical. This is not surprising since the braking strategies of the two algorithms are identical. Besides, both algorithms use the motor during launch to avoid inefficient engine operation. Since the rule-based algorithm is in the charging mode within this time period,
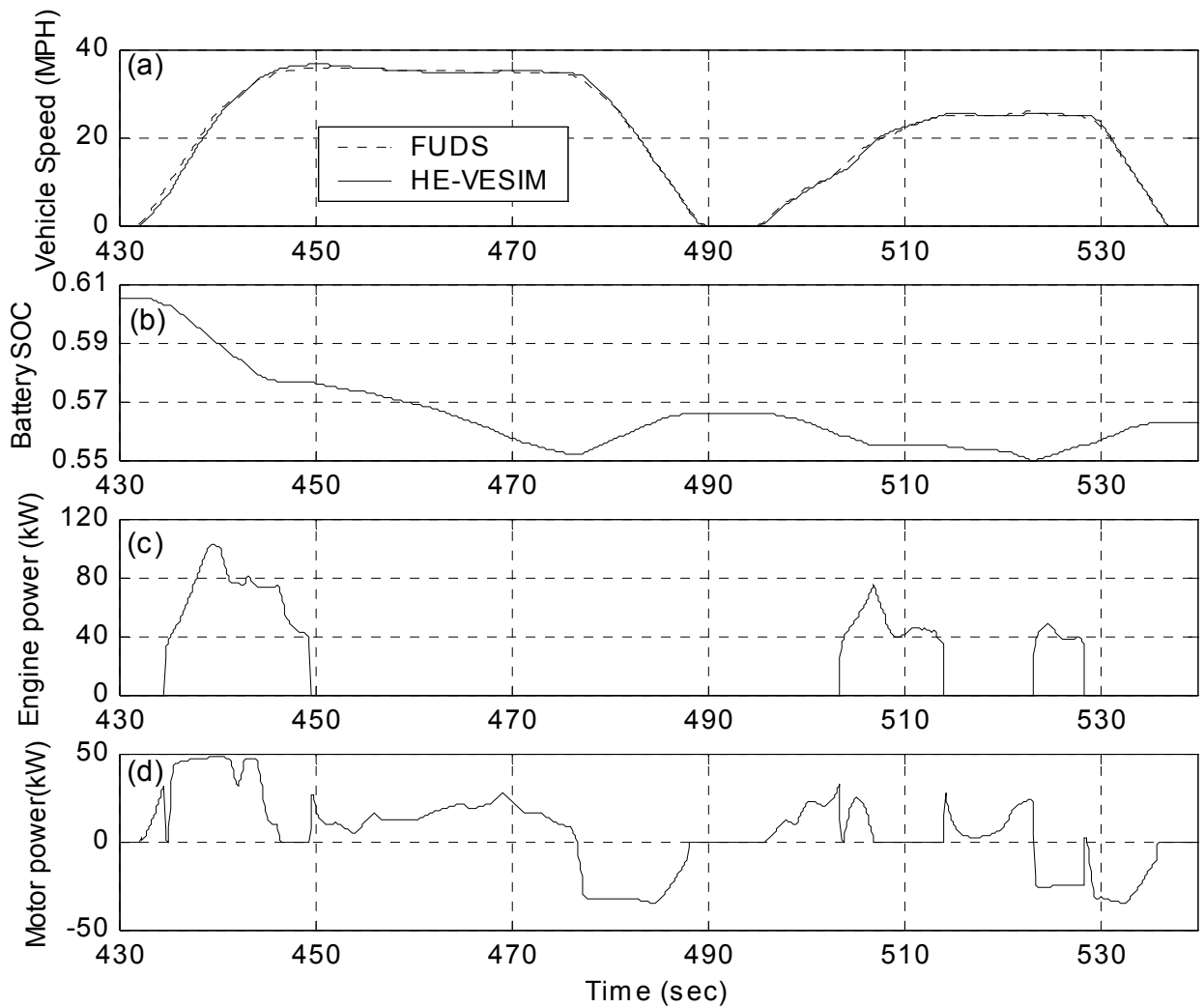

Figure 13: The close-up of the 430 - 540 second interval during the urban driving cycle: a) vehicle speed; b) battery state of charge; c) diesel engine power; and d) electric motor power. 
the battery needs to be recharged whenever the power request is beyond the "engine on" power level until the battery $S O C$ reaches the $S O C_{\text {max }}(60 \%)$. Battery recharge occurs between 698 to $714 \mathrm{sec}$, and 728 to $740 \mathrm{sec}$. Hence, the engine for the rule-based algorithm works harder than that of the dynamic-programming case in order to provide additional constant power $P_{c h}$ to recharge the battery. The fuel economy could suffer. On the contrary, since the dynamic programming optimizes the process over the whole driving cycle, a better discharging/charging schedule has been deployed.

The fuel economy results over the complete urban driving schedule, obtained for the two control algorithms are compared with a conventional diesel engine truck in Table 1. These results are obtained for the charge sustaining strategy, with the SOC at the end of the cycle being the same as it was at the beginning. It can be seen that the fuel economy improvement (over the conventional truck) is about $22 \%$ for the rule-based algorithm and $33 \%$ for the dynamic-programming algorithm. For the case of rule-based algorithm, a large portion of its improvement is due to regenerative braking. Further improvement was obtained by dynamic programming through better-orchestrated coordination between the operation of engine/transmission and motor/battery. Figure 15 shows engine operating points on the BSFC map calculated during the driving schedule for all three vehicle configurations (conventional, HEVrule based and HEV-dynamic programming). The position of the largest clusters of points on both HEV maps is much more favorable compared to the conventional vehicle, i.e. the engine is forced to operate at relatively higher loads and points are moved closer to the high efficiency region. Closer examination of the maps obtained for the two HEV versions indicates that, the rule-based algorithm has achieved a more consistent engine operation near the island of optimum efficiency, but quite a few points are located on the maximum torque line. The dynamic programming algorithm produces higher overall fuel economy by exploring the efficiency of the whole system, instead of focusing just on the engine efficiency. In other words, it appears that in some instances it is more effective to sacrifice some of the engine efficiency and gain on other fronts, through more efficient motor operation and better-optimized charging/discharging schedule. This observation is currently being analyzed in more detail in order to devise an improved rule-based algorithm for the HE-VESIM.

Table 1 Fuel consumption comparison: conventional, dynamic programming (DP), rule-based (RB)

\begin{tabular}{|l|l|l|l|}
\hline & DP & RB & Conventional \\
\hline MPG & 13.85 & 12.65 & 10.39 \\
\hline Fuel (Gallon) & 0.5259 & 0.5757 & 0.7005 \\
\hline
\end{tabular}
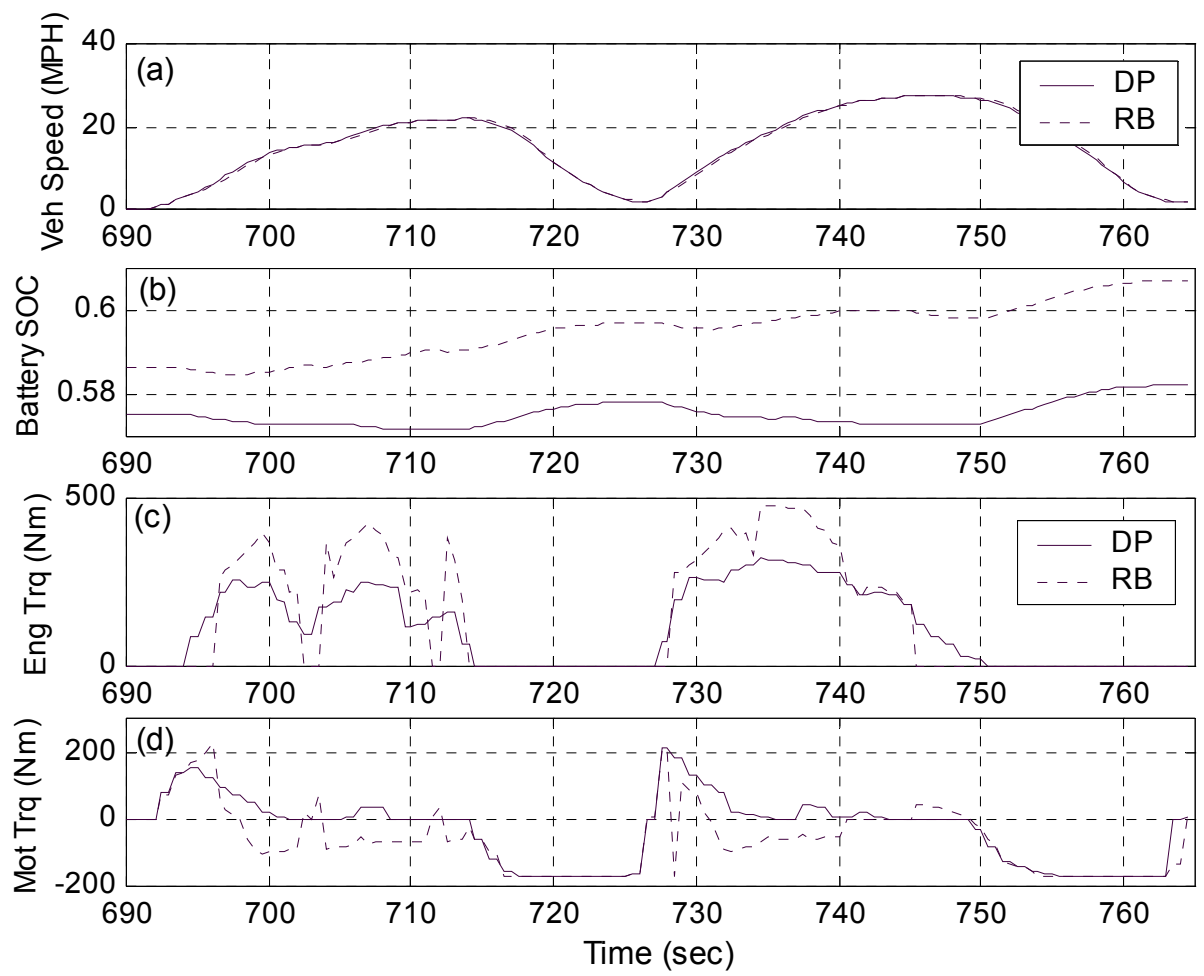

Figure 14: The close-up of the $690-765$ second interval during the urban driving cycle: comparison between the Dynamic Programming (DP) and Rule based (RB) control. 


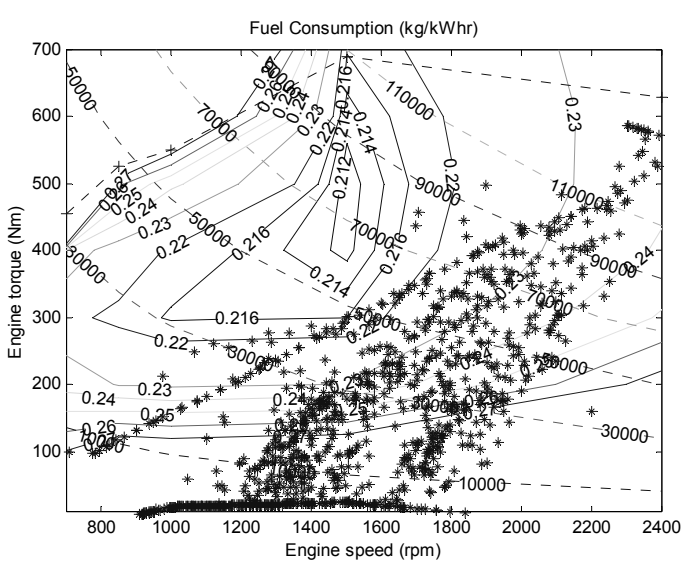

a)

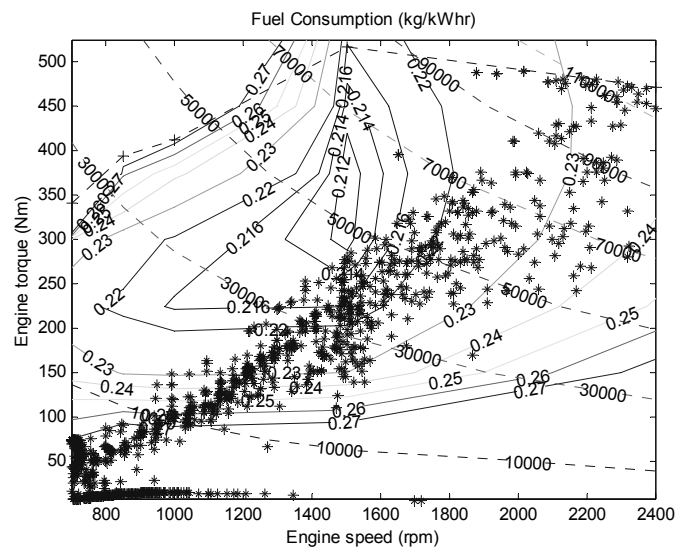

b)

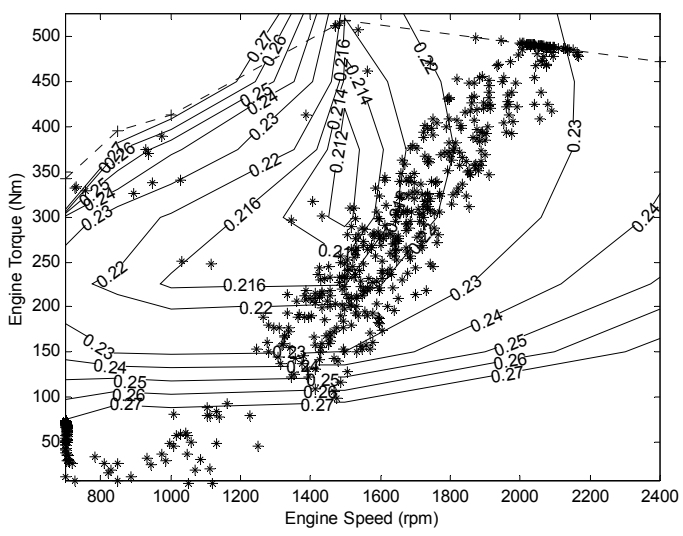

c)

Figure 15: Engine operation comparison over a driving cycle: a) conventional truck; b) hybrid,

\section{SUMMARY AND CONCLUSIONS}

This paper presents the development of a feedforward, parallel, hybrid electric vehicle system simulator, and its use for evaluation of power management strategies aimed at maximizing fuel economy. The simulator is based on a previously validated simulator of conventional engine-vehicle systems that includes modules of the diesel engine, driveline and vehicle dynamics of the appropriate fidelity. The electric components are integrated in the system in the SIMULINK programming environment so that the electric motor/generator is located after the transmission and linked to the output shaft via an electro-mechanical coupling. The diesel engine is downsized since the electric motor is able to provide assistance during the high power demand operation. Two power management algorithms were analyzed in this paper-a dynamic programming algorithm and a rule-based algorithm. In both cases the strategy aims at sustaining the battery state of charge.

The acceleration performance of the HEV Class VI truck was shown to be comparable to the conventional truck. The favorable torque characteristic of the electric motor compensates for the delay in the diesel engine response caused by turbo lag. Simulation of the vehicle over a complete urban driving cycle showed that the fuel economy could be improved by $20-30 \%$ over traditional (non-hybrid) trucks. It was found that the dynamic programming algorithm achieves higher overall fuel economy despite the fact that its engine may often operate in a less efficient region than the one controlled by the rule-based algorithm. This fact illustrates the importance of coordinating multiple inputs, which may not be captured by simple engineering intuition. In other words, in some instances it was more effective to sacrifice some of the engine efficiency, and benefit through more efficient motor operation and optimized charging/discharging schedule, for an overall better compromise.

\section{ACKNOWLEDGMENTS}

The authors would like to acknowledge the technical and financial support of the Automotive Research Center (ARC) by the National Automotive Center (NAC) located within the US Army Tank-Automotive Research, Development and Engineering Center (TARDEC). The ARC is a U.S. Army Center of Excellence for Automotive Research at the University of Michigan, currently in partnership with University of Alaska-Fairbanks, Clemson University, University of lowa, Oakland University, University of Tennessee, Wayne State University, and University of Wisconsin-Madison. The dynamic programming scheme contributions by Jungmo Kang and Jessy Grizzle of the EECS department of The University of Michigan are also gratefully acknowledged.

\section{REFERENCES}

1. Baumann, Bernd M.; Washington, G.; Glenn, Bradley C.; Rizzoni, G., "Mechatronic design and control of hybrid electric vehicles," IEEE/ASME Transactions on Mechatronics, v5 n 1 2000. p 58-72, 2000

2. Farrall, S. D. and Jones, R. P., "Energy management in an automotive electric/hear engine hybrid powertrain using fuzzy decision making." Proceedings of the 1993 International Symposium on Intelligent Control, Chicago, IL, 1993. 
3. Kim, C., NamGoong, E., and Lee, S., "Fuel Economy Optimization for Parallel Hybrid Vehicles with CVT." SAE Paper No. 1999-01-1148.

4. Paganelli, G., Ercole, G., Brahma, A., Guezennec, Y. and Rizzoni, G., "A General Formulation for the Instantaneous Control of the Power Split in ChargeSustaining Hybrid Electric Vehicles." Proceedings of AVEC 2000, 5th Int'l Symposium on Advanced Vehicle Control, Ann Arbor, MI, 2000.

5. Johnson, V.H., Wipke, K.B. and Rausen, D.J., "HEV Control Strategy for Real-Time Optimization of Fuel Economy and Emissions," Proceedings of the Future Car Congress, April 2000, SAE paper\#2000-011543.

6. Brahma, A., Guezennec, Y. and Rizzoni, G., "Dynamic Optimization of Mechanical/Electrical Power Flow in Parallel Hybrid Electric Vehicles" Proceedings of AVEC 2000, 5th Int'I Symposium on Advanced Vehicle Control, Ann Arbor, MI, 2000.

7. Assanis, D.N., Z.S. Filipi, S. Gravante, D. Grohnke, X. Gui, L.S. Louca, G.D. Rideout, J.L. Stein, Y. Wang, 2000. Validation and Use of SIMULINK Integrated, High Fidelity, Engine-In-Vehicle Simulation of the International Class VI Truck. SAE Paper 2000-01-0288, SAE 2000 World Congress.

8. Powell, B.K. and Pilutti, T.E., "A Range Extender Hybrid Electric Vehicle Dynamic Model", Proceedings of the $33^{\text {rd }}$ IEEE Conference on Decision and Control, Lake Buena Vista, FL, December 1994.

9. Burch, Steve, Cuddy, Matt, et. al., "ADVISOR 2.1 Documentation," National Renewable Energy Laboratory, March 1999.

10. Bowles, P. D., "Modeling and Energy Management for a Parallel Hybrid Electric Vehicle (PHEV) with Continuously Variable Transmission (CVT)," MS thesis, University of Michigan, Ann Arbor, MI, 1999.

11. Assanis D.N., and Heywood J.B., Development and Use of a Computer Simulation of the Turbocompounded Diesel System for Engine Performance and Component Heat Transfer Studies," SAE Paper 860329, 1986.

12. Karnopp, D. C., Margolis, D. L., and Rosenberg, R. C., System Dynamics: A Unified Approach. Wiley-Interscience, New York, New York, 1990.

13. Rosenberg, R. C., and Karnopp, D. C., Introduction to Physical System Dynamics. McGraw-Hill, New York, New York, 1983

14. 20SIM, 20SIM Pro Users' Manual. The University of Twente - Controllab Products B.V. Enschede, The Netherlands, 1999
15. Louca, L. S., Stein, J. L., Hulbert, G. M., "A PhysicalBased Model Reduction Metric with an Application to Vehicle Dynamics". The 4th IFAC Nonlinear Control Systems Design Symposium (NOLCOS 98). Enschede, The Netherlands, 1998.

16. Louca, L.S., J.L. Stein and D.G. Rideout, 2001. Generating Proper Integrated Dynamic Models for Vehicle Mobility Using a Bond Graph Formulation. Proceedings of the 2001 International Conference on Bond Graph Modeling, January, Phoenix, AZ. Published by the Society for Computer Simulation.

17. Liu, H., Chalhoub, N. G., Henein, N., "Simulation of a Single Cylinder Diesel Engine Under Cold Start Conditions Using SIMULINK", Proceedings of ASME-ICE Spring Technical Conference, Vol. 28-1, Fort Collins, CO, April 27-30, 1997.

18. Wiegman, H. L. N., Vandenput, A. J. A., "Battery State Control Techniques for Charge Sustaining Applications," SAE Paper 981129, 1998.

19. Jun-Mo Kang, Ilya Kolmanovsky and J.W. Grizzle "Approximate Dynamic Programming Solutions for Lean Burn Engine Aftertreatment," Proceeding of the IEEE Conference on Decision and Control, Phoenix, AZ, December 7-10, 1999.

20. Assanis D., Delagrammatikas, G, Fellini, R., Filipi, Z., Liedtke, J., Michelena, N., Papalambros, P., Reyes, D., Rosenbaum, D., Sales, A., Sasena, M.,"An Optimization Approach to Hybrid Electric Propulsion System Design", Mechanics of Structures and Machines, Volume 27, No. 4, 1999, pp. 393 421

\section{APENDIX}


Table 1: DI Diesel Engine Specification

\begin{tabular}{|l|l|l|}
\hline & CONVENTIONAL & HYBRID \\
\hline Configuration & $\begin{array}{l}\text { V8, Turbocharged, } \\
\text { Intercooled }\end{array}$ & $\begin{array}{l}\text { V6, Turbocharged, } \\
\text { Intercooled }\end{array}$ \\
\hline Displacement [L] & 7.3 & 5.475 \\
\hline Bore [cm] & 10.44 & 10.44 \\
\hline Stroke [cm] & 10.62 & 10.62 \\
\hline Con. Rod Length [cm] & 18.11 & 18.11 \\
\hline Compression Ratio [-] & 17.4 & 17.4 \\
\hline Rated Power [HP] & 210 @ 2400 rpm & $157 @ 2400 \mathrm{rpm}$ \\
\hline
\end{tabular}

Table 2: Drivetrain Specifications

\begin{tabular}{|l|l|}
\hline Torque converter - Turbine inertia [kg ${ }^{*} \mathrm{~m}^{2}$ ] & 0.068 \\
\hline Transmission - 1st gear churning losses coeff. R11 & 0.0192 \\
\hline Transmission - 2nd gear churning losses coeff. R12 & 0.015 \\
\hline Transmission - 3rd gear churning losses coeff.R13 & 0.031 \\
\hline Transmission - 4th gear churning losses coeff.R14 & 0.0367 \\
\hline Transmission - 1st gear churning losses coeff.R21 & $1.36110^{-5}$ \\
\hline Transmission - 2nd gear churning losses coeff.R22 & $5.71910^{-6}$ \\
\hline Transmission - 3rd gear churning losses coeff.R23 & $-3.18910^{-5}$ \\
\hline Transmission - 4th gear churning losses coeff.R24 & $-4.17710^{-5}$ \\
\hline Transmission - 1st gear ratio [-] & 3.45 \\
\hline Transmission - 2nd gear ratio [-] & 2.24 \\
\hline Transmission - 3rd gear ratio [-] & 1.41 \\
\hline Transmission - 4th gear ratio [-] & 1.00 \\
\hline Transmission - 1st gear ratio [-] FOR HYBRID & 2.59 \\
\hline Transmission - 2nd gear ratio [-] FOR HYBRID & 1.68 \\
\hline Transmission - 3rd gear ratio [-] FOR HYBRID & 1.06 \\
\hline Transmission - 4th gear ratio [-] FOR HYBRID & 0.75 \\
\hline Transmission - Fluid charging pump loss [Nm] & -6.12 \\
\hline Transmission - 1st Gear efficiency [-] & 0.9893 \\
\hline Transmission - 2nd Gear efficiency [-] & 0.966 \\
\hline Transmission - 3rd Gear efficiency [-] & 0.9957 \\
\hline Transmission - 4th Gear efficiency [-] & 1.0 \\
\hline Propshafts/Differential - Axle churning loss coeff.R0 & 8.34 \\
\hline Propshafts/Differential - Axle churning loss coeff.R1 & 0.04087 \\
\hline Propshafts/Differential - Differential drive ratio [-] & 3.21 \\
\hline Propshafts/Differential - Differential efficiency [-] & 0.96 \\
\hline
\end{tabular}

Table 3: Vehicle Dynamics Specifications

\begin{tabular}{|c|c|}
\hline CG location from front axle [-] & 0.61875 \\
\hline Sprung mass [kg] & 6581.6 \\
\hline Unsprung mass rear $[\mathrm{kg}]$ & 430.9 \\
\hline Unsprung mass front $[\mathrm{kg}]$ & 244.9 \\
\hline Longitudinal - Wheel inertia $\left[\mathrm{kg}^{\star} \mathrm{m}^{2}\right]$ & 18.755 \\
\hline Longitudinal - Break viscous damping $\left[\mathrm{N}^{*} \mathrm{~m}^{*} \mathrm{~s} / \mathrm{rad}\right]$ & 100.0 \\
\hline Longitudinal - Break coulomb damping [ $\left.\mathrm{N}^{*} \mathrm{~m}\right]$ & 0.0 \\
\hline Longitudinal - Wheel radius [m] & 0.4131 \\
\hline Longitudinal - Tire pressure [psi] & 115.0 \\
\hline Longitudinal - Number of tires on rear axle [-] & 4.0 \\
\hline Longitudinal - Wheel bearing damping $\left[\mathrm{N}^{*} \mathrm{~m}^{*} \mathrm{~s} / \mathrm{rad}\right]$ & 3.0 \\
\hline Longitudinal - Road/tire friction coefficient [-] & 0.7 \\
\hline Longitudinal- Aerodynamic drag $=0.5^{*} \mathrm{Cd}^{*} \rho^{\star}$ Area & 2.081 \\
\hline Vertical - Rear suspension compliance [m/N] & $6.3446110^{-7}$ \\
\hline Vertical - Rear tire compliance [m/N] & $2.9740310^{-7}$ \\
\hline Vertical - Rear suspension damping $\left[\mathrm{N}^{*} \mathrm{~s} / \mathrm{m}\right]$ & 7000.0 \\
\hline Vertical - Rear tire damping $\left[N^{*} \mathrm{~s} / \mathrm{m}\right]$ & 2000.0 \\
\hline
\end{tabular}

